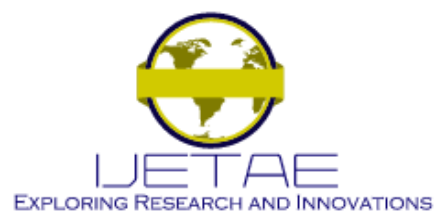

International Journal of Emerging Technology and Advanced Engineering Website: www.ijetae.com (ISSN 2250-2459, ISO 9001:2008 Certified Journal, Volume 10, Issue 07, July 2020)

\title{
A Functional Safety Methodology Based on IEC 61508 for Critical Reliability FPGA-Based Designs
}

\author{
Bruna F. Flesch ${ }^{1}$, Igor Tedeschi ${ }^{2}$, Rodrigo Marques de Figueiredo ${ }^{3}$, Lúcio Rene Prade ${ }^{4}$, Márcio Rosa da Silva ${ }^{5}$ \\ 1,2,3,4,5 Polytechnic School \& 950 Unisinos ave - São Leopoldo / RS - Brazil
}

\begin{abstract}
Field Programmable Gate Arrays (FPGAs) have been more and more used in functional safety designs. Commonly, such projects present high - or ultra high complexity, which might increase susceptibility to meta instability issues. Regardless, they are usually exposed to severe environmental conditions, so that evaluating their safety life cycle is an important tool in order to acquire the targeted safety level. So, this research studies a functional safety methodology based on the V-model, which is described in the international standard IEC 61508-2 (2010). Such model suggests both verification and validation of each stage that constitutes the analysed application, whose steps are specification of safety requirements, architecture definition, conceptual design and detailed design, specification of synthesis parameters, coding, testing (unitary, modular and integration) and documentation. Fault analysis was developed by using the formal method Failure Mode and Effect Analysis (FMEA). From the results, it might be stated that technique lockstep and 2-1 line multiplexer with a three-bit selector, which was based on the 1003 architecture from the standard IEC 615082 (2010), enabled addressing higher safe failure fraction (SFF) than by using 2003 architecture with a majority voter. This leads to higher safety integrity levels (SILs), so that a SIL 3 might be addressed in programmable electronic. By using a IEC61508-5 (2010) quantitative measure to determinate SIL, it might be stated that following components presented a SIL 1: logic gates and, or, nand and not, flip-flop $D$ and a two bit counter. Triple Modular Redundancy (TMR) technique showed a SIL 2 level. Both lockstep and three bit selector multiplexer presented a SIL 3 and a $75 \%$ SFF.
\end{abstract}

Keywords - IEC 61508, field programmable gate arrays, functional safety methodology, reliability-critical applications, Vmodel.

\section{INTRODUCTION}

To begin with, according to [1], SRAM-based field programmable gate arrays (FPGAs) have been more and more used to create high complex circuits in the last decade due to reduced development cost of programmable logic, high flexibility and associated low cost. In fact, there is great interest in their use for non-invasive medical instrumentation [2], readout electronics toward a radiation environment [3], smart system for controlling of electrical power generation plants [4], high-altitude environment and reliability-critical terrestrial-based applications [5]. In spite of this, a safety methodology was proposed to be applied in fault tolerant FPGA designs in [6].
With regards to fault-mode, it is well known that device shrinkage increases vulnerability toward charged particles that commonly strike them [5]. It should also be mentioned that external factors, such as pressure and temperature may also produce errors in combinational and sequential elements. Nevertheless, high complex circuits usually contain both increased cross-sections and large number of asynchronous clocks. In such designs, there might be signals being transferred between circuits with unrelated clocks domains. In this case, timing violations may occur, leading to a metastability failure. Metastability might not be completely avoided concerning reliability of digital design. In that case it is important evaluation of both failure and moment of occurrence [7].

So, [1] states that increasing protection of Finite State Machines (FSMs) is needed based on the fact that they control the overall system functioning. By applying the methodology proposed in this research, it will be possible increasing protection of programmable logic FSMs and defining safe states to which a system will be driven to in case a fault matter. The referred methodology is mainly formed by application of V-model presented in Figure I, definition of system requirements and specifications, identification of safety methods to be used, implementation, testing (unit, integrity and functional) and report evaluation.

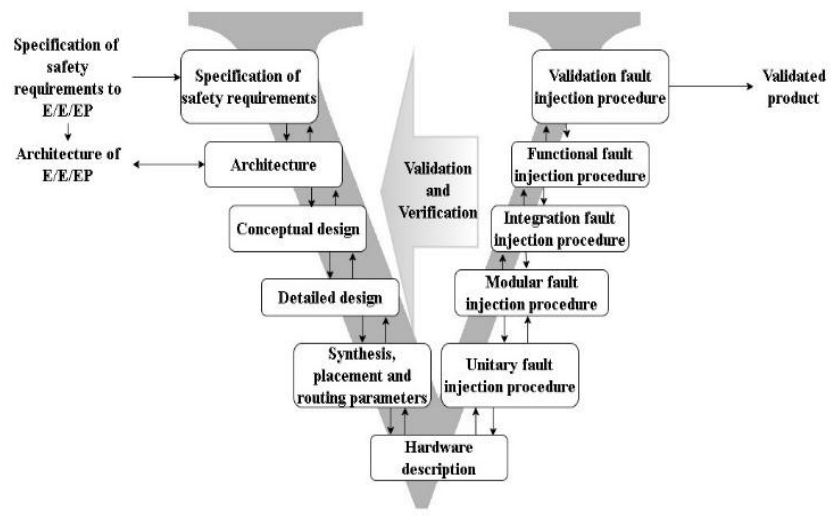

FIGURE I V-MODEL 


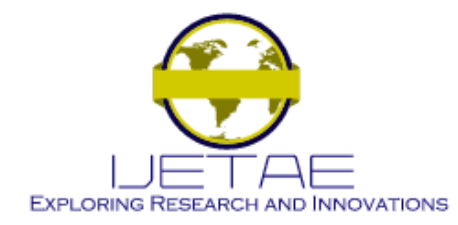

\section{International Journal of Emerging Technology and Advanced Engineering Website: www.ijetae.com (ISSN 2250-2459, ISO 9001:2008 Certified Journal, Volume 10, Issue 07, July 2020)}

This work is organized as follows: the second section presents fault modes related to FPGA-based designs. The third section focuses on the state-of-the-art. The methodological proposal is addressed in the fourth section. The case study in which such methodology was applied is explained in the fifth section, followed by results discussion and conclusion.

\section{FAult Modes}

In the study of [8], it has been stated that fault modes of a system or process are characterized as all events capable of leading an asset to fail.

Furthermore, such fault modes may be related to probable causes of functional failures. In fact, they involve partial or complete reduction of a product functioning rate and performance goals. Then, identifying fault modes of systems and subsystems presents significant relevance towards an accurate evaluation not only of reliability, but also to availability, functional safety, confidentiality, integrity and maintenance.

In the light of these facts, identifying fault causes and their mechanisms enables evaluation of fault modes [8], which contain functioning effects and quality.

It has been stated that all aspects of FPGA-based designs are very sensitive to terrestrial-based radiation, being particularly sensitive to neutron radiation [5]. Nevertheless, they require large amount of silicon in order to establish infield reconfigurability [9]. Following this discussion, such reconfigurability might lead to a 35 times rising of a design silicon area as well as it may increase critical path delays by 3 to 4 times [10].

In spite of SRAM-based configuration cells affected by charged particles, [9] explains that upsets within the configuration memory are increasing. This fact is due to the expanding number of configuration cells used in a target. Indeed, such cells are responsible for routing, operation of the logic [9] among other tasks. So, an error in their data might compromise not only these but other system routines. However, [5] states that FPGAs are susceptible not only to soft error induced state but to configuration changes as well.

The explanation about how physics, system designs and environment contribute to raise soft error occurrence is developed in [11], [5]. The first aspect relies on the fact that a smaller transistor is more sensitive to a smaller electromagnetic charge. Also, design characteristics might lead to cross sections increase.
It is also quoted that high-altitude military applications are more than 300 times more exposed to neutron radiation than in sea level [5], exemplifying how much an environment may impact on soft error occurrence in FPGA-based designs.

A large spectrum of Single Event Effects (SEEs) might be produced by the occurrence of Single Event Upsets (SEU) in FPGA memory cells, such as configuration memory, block memory, distributed memory, flip-flops and internal device control [12]. The same study highlights that a long latency related to memory cells implies in a late change of the systems output. Sometimes, such errors may be even harmless due to the large amount of routing and logic configuration bits unused in a circuit and long latency of memory cells.

Furthermore, impacts of exposure to high-energy ionizing radiation are addressed in [9] and [13]. Such studies explain how it changes internal structure of MOS transistors, threshold voltage changing, transient currents increasing and electrical characteristics. In the short time, these SEEs are produced by Single Event Upsets (SEUs), Single Event Transients (SETs), Single Event Latchups (SELs) and Single Event Functional Interrupts (SEFIs) [9].

Regarding metastability failures, they should also be investigated when evaluating different fault modes of reliability critical FPGA projects. In fact, such phenomena are related to synchronization of two or more signals and might occur more often in high complex designs containing several clock domains.

Basically, a metastability failure takes place when data and clock do not respect setup and hold timing specifications [7]. An easy way to minimize this issue is by using D-type flip-flops in order to register outputs of a logic block.

The most basic fault mode of a digital system is a single bit fault. Firstly, it might erroneously be stucked at a logical level. Usually, these faults are related to long paths, single way connections, fan networks, congested interconnections [14], manufacturing problems, usage time [15]. Secondly, it is possible the occurrence of a bit-flip, described in [16], [17]. Equally important are fault modes of digital components such as:

A. logic gates: [18] states that their internal fault modes are open fault $(\mathrm{OF})$ which occurs in I/Os and electrical nodes and bridging fault in pairs of electrical nodes; 


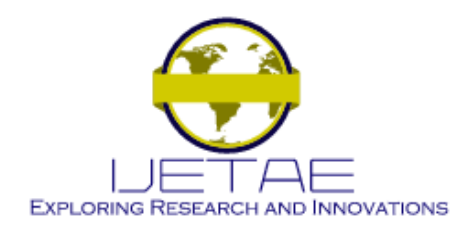

International Journal of Emerging Technology and Advanced Engineering

Website: www.ijetae.com (ISSN 2250-2459, ISO 9001:2008 Certified Journal, Volume 10, Issue 07, July 2020)

B. flip-flops: there have been several studies addressing fault tolerant flip-flops [19], [20], [21], [22]. An important part of functional safety designs is that it requires hardware redundancy, according to IEC 61508;

C. finite state machines (FSMs): they are studied in [23], [1]. They need to be safe because are responsible for controlling general functioning of a system. Faults occurring in clock signals or state variables might compromise accurate behavior of a FSM.

\section{STATE-OF-THE-ART}

Recent works have been published about this subject. A soft error tolerant FPGA architecture is proposed in [24]. It is based on double modular redundancy (DMR) error correction circuit and is able to mitigate multiple bit upsets (MBUs). Results of this paper have shown that the referred architecture could handle MBUs by using a smaller area than designs containing error correction codes (ECCs) or triple modular redundancy (TMR).

In [25], a modified matrix code (MMC) is applied to correction of MBUs in FPGA-based designs. They also used dynamic partial reconfigurability to reduce reconfiguration timing. [26] explains that Xilinx has been working for more than 15 years in order to ensure usage of its products in critical applications. It also states that ISE Design Suite 14.2-14.7 received certification for IEC 61508 and ISO 26262. The same company developed material returning procedures, and a production process focused on prevention of quality issues. Such process uses cause and effect analysis to maximize reliability, availability and quality of its products.

In the study developed in [9], FPGA-based designs with high reliability are addressed. Radiation effects, radioactive environments, architecture vulnerabilities, SEE mitigation techniques are studied with focus on fault tolerance. In a like manner, a method for analyzing hidden faults in modern safety-related systems is evaluated in [27], [28], [29].

Regarding the lockstep technique, it is used in [30] to enhance functional safety in FPGA-based motor drives. However, functional metrics are not evaluated in such study. Lockstep is also cited in [31] to specify the architecture of the microcontroller family RM48x commonly used in safety applications.

Hence, [31] explains that power circuit, clocks, reset and basic processing functions contain a diagnostic subsystem in hardware in high level of abstraction. A lockstep approach with two kernels detects errors in kernel neighborhood once at a clock cycle.
There has been a huge number of studies addressing fault tolerance [32], [1], [33], [34], [35], [36] and hardware robustness.

Although the number of researches focusing in functional safety is much smaller, it is expected to increase continuously. [37] implemented two computational models to facilitate decision taking of the required SIL level for a SIS. Furthermore, [38] human and organizational factors are taken into account to calculate SIL level.

With relation to fault injection technique, which is mandatory according [39], [36] uses it to validate data transmitting of a safety communication protocol.

Design tools were evaluated by [40] in order to establish redundant systems with only a single chip die. Fault insertion testing for PLC-based system SIL certification is studied in [41].

A description of implementation and integration process of a complete FPGA-based communication system with use of 1002 architecture is performed in [33]. The same work explains that a diagnostic subsystem detects software and hardware faults in the main system and then activates a redundant one free of faults.

On-chip hardware redundancy is proposed in [42] to accomplish the second edition of IEC 61508.

Hardware Description Language (HDL) Designs are considered to safety critical applications in nuclear power plants in [43]. It proposes an architecture of a formal verification method to be used with HDLs such as VHDL or Verilog.

In [44], two 32-bit controllers are used to mask a fault mode. It is acquired through CPU automatic tests, watchdog and also by using a SEU mitigation controller developed by Xilinx.

\section{PROPOSED METHODOLOGY}

This work relies on the application of architectures 1003 and 2003 and the V-model proposed in [45]. [38] explains that vulnerabilities may occur in a system for active and latent faults. In addition, the same work elucidates that active faults are expressed as unsafe actions taken by users whereas latent ones are inherent in a system. In fact, using the V-model enables dealing with latent faults, that is, weaknesses of an embedded design.

To begin with, the developing a conceptual test was to verify whether this methodology could be applied in embedded systems described in programmable logic. As a conceptual test, its application was defined as simple as possible. 


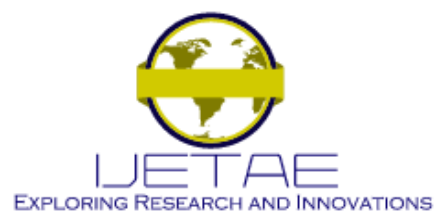

\section{International Journal of Emerging Technology and Advanced Engineering Website: www.ijetae.com (ISSN 2250-2459, ISO 9001:2008 Certified Journal, Volume 10, Issue 07, July 2020)}

Then, safety requirements were specified so that architecture, conceptual and detailed project could be described.

Thirdly, hardware descriptions were written in VHDL. From here on, fault injection procedures (unitary, modular and integration ones) were specified and implemented. These stages are presented in next sections.

\section{A. Conceptual test}

The conceptual test consists of a simple Moore FSM with four states described in VHDL. Both lockstep and TMR were applied on it. Thus, state variables of all three FSM redundant domains were connected both to a comparator and a majority voter. Because of the comparator, whenever a fault strikes state variables leaving them to different values, all three redundant domains were driven to the highest priority state, called safe state (State $0)$. Also, the 2003 architecture with a majority voter is applied to output logic circuits. Consequently, a single fault striking a FSM output would not affect such signal.

Figure II presents the proposed system.

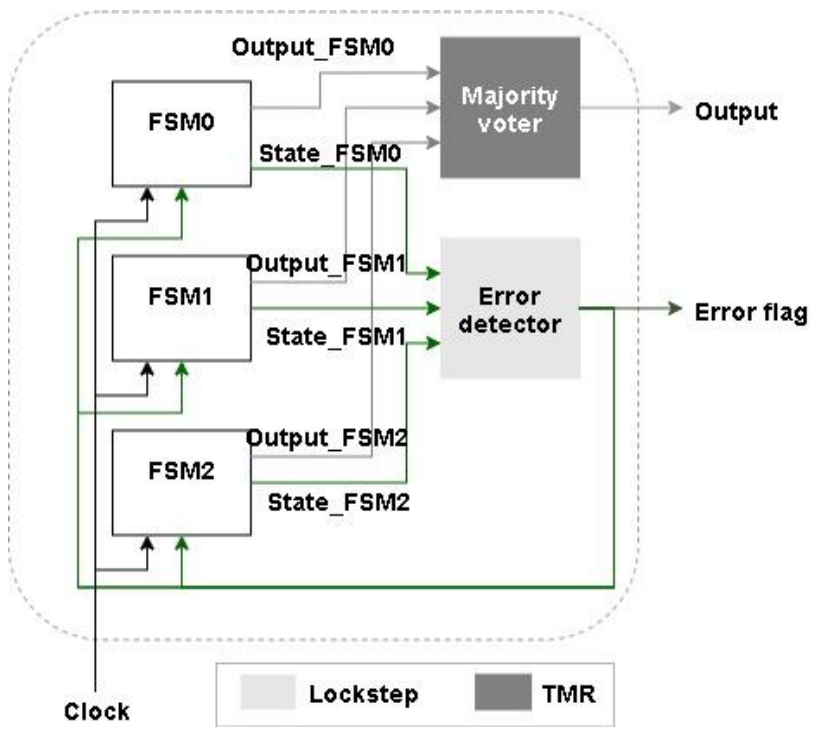

\section{FIGURE II CONCEPTUAL TEST EVALUATED}

Regarding Figure II, it should be mentioned that all output modules were registered to facilitate synchronization as recommended in IEC61508-2:2010. Furthermore, the same standard contains a safety lifecycle proposal, which should be considered in hardware projects aiming to accomplish a SIL level. By doing it, the referred system might be able to handle random and systematic failures.
Specifically, clause 7.1.3.1 of the standard advises usage of the V-model, in which there is a corresponding verification and validation for each stage of development process. Equally important, it is also relevant to mention that the design method should be in accordance with the targeted SIL level.

\section{B. Safety requirements}

Safety requirement specification refer to specification of Safety Instrumented Functions (SIFs) to be applied in conceptual test by using Failure Mode and Effect Analysis (FMEA). It correlates hierarchy, safety requirement, fault modes, fault causes, probability of occurrence, prevention activities and testing. Besides, multiplexers used in this study were described in [6] and present 1003 architecture from [46].

Fault analysis of the conceptual test was implemented through evaluation of Register Transfer Level file (RTL) files of each VHDL module implemented.

It should also be mentioned that monitored redundancy was implemented with use of lockstep, whose purpose is comparing current state of the three redundant domains and driving the FSM to a safe state in case of a fault. The safe state is State 0 .

From the FMEA evaluation, a safety plan was defined. Also, it should be mentioned that, accordingly to Annex A of [39], the safety plan of a system has to contain safety metrics to its evaluation.

\section{Safety plan}

In order to avoid faults during development of this research, requirements tracking was applied by using FMEA. Nevertheless, in respect to the project life cycle, fault injection procedures described there were performed by different person.

A safety state was defined to acquire fault avoidance measures. They also include use of Xilinx ISE Design Suite 14.7 which is certified to IEC 61508-3, as well as application of a management and automation fault injection tool.

System modeling were addressed with flowcharts ([39] Annex B Table B.5), use of FSMs which is recommended to SIL 2, SIL 3 and SIL 4 applications. Modification control was performed with impact analysis tool, recheck of altered modules and those affected by the changes made, as IEC61508-3:2010 Annex A guidelines, Table A.8.

Accordingly to Annex B of [39], semi-formal methods applied are: function block diagrams; transition state diagrams and truth tables. 


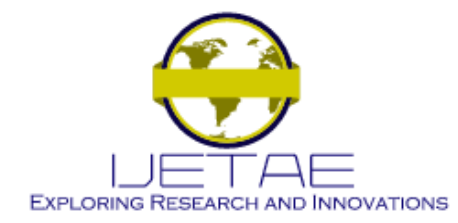

International Journal of Emerging Technology and Advanced Engineering

Website: www.ijetae.com (ISSN 2250-2459, ISO 9001:2008 Certified Journal, Volume 10, Issue 07, July 2020)

Equally important is the use of majority voter, monitored redundancy with lockstep, structured specification and inspection, structural project organization and modularization as well as synthesis parameters specification. These techniques are in accordance to Annex $\mathrm{A}$ and $\mathrm{B}$ of [39] for addressing faults occurring during a project safety life.

\section{RESUlTS AND DisCUSSION}

All modules were tested individually once. Also, ten fault injection procedures were performed to the complete system, whose scope is similar to the one applied in individual modules and is listed below:

- $\quad$ Simulation total length: 84,00075 $\mu \mathrm{s}$;

- Total number of inserted faults: 2000 ;

- Fault period: 42 ns;

- Duty cycle of each fault: $25 \%$;

- Clock period: $10 \mathrm{~ns}$;

- Selection of signals to receive faults: random;

- Fault value: it varies in order to ensure that a fault value is different of a signal original value.

- Clock period of inspection: $1 \mathrm{~ns}$.

Failure rates were calculated from metrics presented in [47]. Figures 3 and 4 presents results from fault injection procedures and reliability calculated with equation 1 [48].

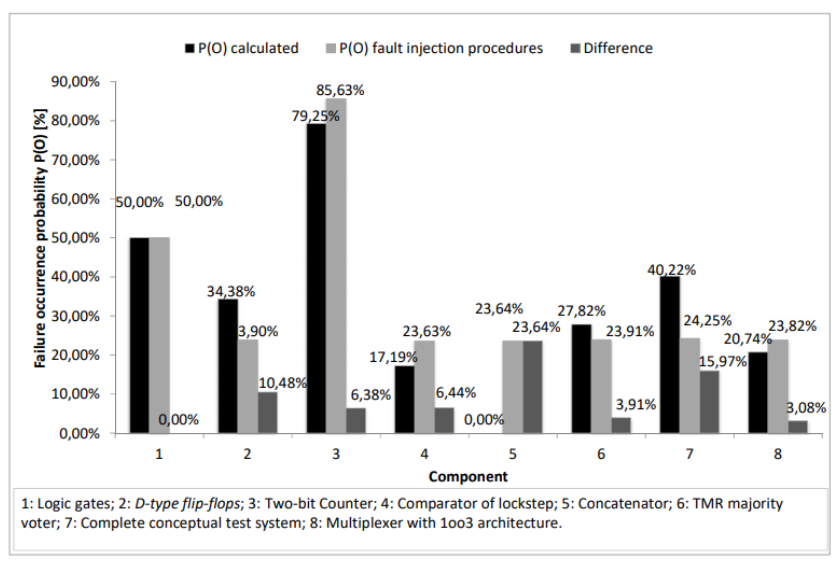

\section{FIGURE III FAILURE OCCURRENCE PROBABILITY FOR SUBSYSTEMS} AND FOR THE COMPLETE CONCEPTUAL TEST

Figure III enables stating that both subsystems and the complete system present failure occurrence probabilities in accordance to calculated values, excepting subsystem five.

It might be related to the fact that such component contain primitives, whose internal structure may not be seen.

$$
C(t)=\int_{t}^{\infty} f(t) d t=1-\int_{t}^{\infty} f(t) d t=1-F(t)
$$

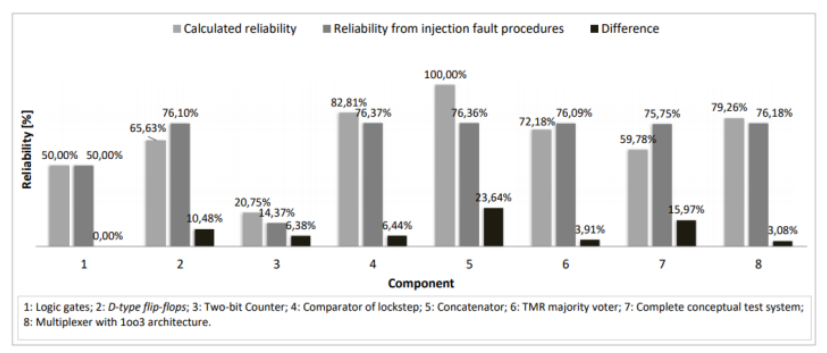

\section{FIGURE IV RELIABILITY OF SUBSYSTEMS AND COMPLETE CONCEPTUAL TEST}

It should also be mentioned that, from Figure 4, components four and five are critical from the system reliability, because both of them are not redundant.

Diagnostic coverage was calculated by using equation 2 [45]. $\lambda_{\mathrm{DD}}$ is dangerous failure detected and $\lambda_{\mathrm{D}}$ refers to dangerous failure.

$$
D C=\frac{\sum \lambda_{D D}}{\sum \lambda_{D}}
$$

There are five different approaches to calculate a SIL level as mentioned in Annex B of [49]. This study focused on the quantitative method. It requires calculation of safe failure detected $\lambda_{\mathrm{SD}}$, safe failure undetected $\lambda_{\mathrm{SU}}$, safe failure $\lambda_{S}$, safe failure fraction SFF and failure rate $\lambda$. The equation 3 was applied to calculate failure rate with $\mathrm{P}(\mathrm{O})$ from fault injection procedures.

$$
\lambda=\frac{P(O) * f_{\text {total }}}{720 \text { hours }}
$$

Safe failure fraction was calculated with equation 4.

$$
S F F=\frac{\sum \lambda_{S}+\sum \lambda_{D D}}{\sum \lambda_{S}+\sum \lambda_{D D}+\sum \lambda_{D U}}
$$

Hardware Fault Tolerance (HFT) in Table I depends on the MooN safety architecture.

TABLE I

SFF, HFT AND SIL BY COMPONENT

\begin{tabular}{|l|c|c|c|}
\hline Component & SFF [\%] & HFT & SIL level \\
\hline Logic gates & 0,00 & 0 & 1 \\
\hline D-type Flip-flops & 0,00 & 0 & 1 \\
\hline Two-bit counter & 0,00 & 0 & 1 \\
\hline $\begin{array}{l}\text { Lockstep } \\
\text { comparator }\end{array}$ & 75,0 & 2 & 3 \\
\hline Concatenator & 0,00 & 0 & 1 \\
\hline TMR Voter & 18,5 & 1 & 2 \\
\hline 1oo3 Multiplexer & 75,0 & 2 & 3 \\
\hline
\end{tabular}




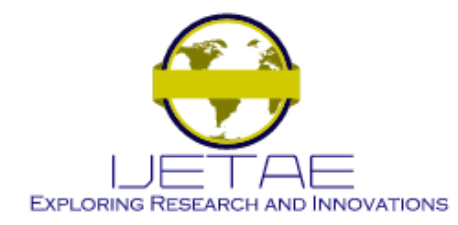

\section{International Journal of Emerging Technology and Advanced Engineering}

Website: www.ijetae.com (ISSN 2250-2459, ISO 9001:2008 Certified Journal, Volume 10, Issue 07, July 2020)

From Table I, it might be stated that lockstep allowed acquisition of a higher SFF even though the system was under influence of 500 faults to a $20 \mu$ s simulation.

With regard to lockstep and 1003 multiplexer, it has to be stated that programmable logic reduced SIL from 4 to 3. Nevertheless, the complete system presents a SIL 2 level.

The final step of this research was to establish a list of data describing how its documentation is organized. Such measure acquires [50], which states that every stage of a safety project should present, at least: safety plan verification plan, verification report, functional safety evaluation plan and functional safety evaluation report.

\section{CONCLUSION}

This study focused on the application of a functional safety methodology towards SRAM FPGA-based designs. It should also be mentioned that even nowadays the use of FPGA in Safety Instrumented System (SIS) might be considered with apprehension.

Regarding tools and methods used in this work, they were based on the guidelines of the Volumes 1 to 7 of IEC 61508 (2010) standard, from the application of formal methods as FMEA, to the semi-formal ones, as state transition diagrams, FSM usage and truth tables.

All V-model stages and evaluations performed in this research were functional. By using IEC 61508 it was possible to implement a bottom-up approach design containing fault modes, specification of functional safety evaluations and metrics, automatic fault injection procedure and identification of calculation of SIL level.

SIL levels calculated enable to state that both lockstep and 1003 multiplexer reached a higher SFF than by using TMR. In fact, their SIL level is 3, whereas TMR presents a SIL 2.

Future areas of study, applying this methodology in a real application. Also, it is certainly relevant to compare different dependability evaluation tools, such as Worst Case Analysis, Expanded Functional Tests and Worst Case Test.

From the aforementioned, this work intends to propose a first approach in order to apply functional safety in programmable logic designs, as well as to compare quantitatively different functional safety architectures. So, it might be said that it may be used as an implementation tool toward more complex safety critical programmable logic designs.

\section{REFERENCES}

[1] L. Frigerio and F. Salice, "Ram-based fault tolerant state machines for fpgas," in 22nd IEEE International Symposium on Defect and Fault Tolerance in VLSI Systems, Sept 2007, pp. 312-320.

[2] M. Riyadi, I. Iskandar, and A. Rizal, "Development of FPGA-based threelead electrocardiography," in 2016 International Seminar on Intelligent Technology and Its Application, 2016, pp. 67-72.

[3] J. e. a. Alme, "Radiation-tolerant, SRAM-FPGA based trigger and readout electronics for the ALICE experiment," in IEEE TRANSACTIONS ON NUCLEAR SCIENCE, vol. 55, no. 1, February 2008, pp. 76-83.

[4] B. Siswoyo, "FPGA-based smart system architectural design for characterizing and controlling wind turbine in electrical power generation," in 2016 International Seminar on Intelligent Technology and Its Applications (ISITIA), 2016, pp. 479-486.

[5] H. Quinn and P. Graham, "Terrestrial-based radiation upsets: A cautionary tale," in 13th Annual IEEE Symposium on FieldProgrammable Custom Computing Machines (FCCM'05), 2005, pp. 193-202.

[6] B. Flesch, B. Brand, R. M. de Figueiredo, L. R. Prade, and M. R. da Silva, "Proposal of a functional safety methodology applied to fault tolerance in FPGA applications," in 17th IEEE Latin-American Test Symposium - LATS 2016, April 2016, pp. 8-13.

[7] B. Rogina and P. Skoda, "Metastability testing at FPGA circuit design using propagation time characterization," in Design \& Test Symposium, Sept 2010, pp. 1-6.

[8] F. Fogliatto, "Manutenção centrada em confiabilidade (MCC)," 2017, Available in: <http://www.lume.ufrgs.br/bitstream/handle/10183/29050/00077431 2.pdf/>.

[9] M. Wirthlin, "High-reliability FPGA-based systems: Space, highenergy physics, and beyond," in Proc. of the IEEE, vol. 103, no. 3, March 2015, pp. 379-389.

[10] I. Kuon and J. Rose, "Measuring the gap between FPGAs and ASICs," in IEEE TRANSACTIONS ON COMPUTER-AIDED DESIGN OF INTEGRATED CIRCUITS AND SYSTEMS, vol. 26, no. 2, Feb 2007, pp. 203-216.

[11] J. Azambuja, S. Pagliarini, M. Altieri, F. Kastensmidt, M. Hubner, and J. Becker, "A fault tolerant approach to detect transient faults in microprocessors based on a non-intrusive reconfigurable hardware," in IEEE Transactions on Nuclear Science, 2012, pp. 1117-1124.

[12] J. L. Nunes, J. C. Cunha, and M. Zenha-Rela, "On the effects of cumulative SEUs in FPGA-based systems," in 12th European Dependable Computing Conference, Dec 2016, pp. 89-96.

[13] H. Sandberg, "Radiation hardened system design with mitigation and detection in FPGA," Master of Science Thesis in Electrical Engineering, Department of Electrical Engineering, Linkoping University, Linkoping, Sweden, 2016.

[14] O. Patino and J. Martinez-Santos, "Physical-aware pattern selection for stuck-at faults," in 2017 18th IEEE Latin American Test Symposium (LATS), 2017, pp. 1-5.

[15] B. Bhowmik, J. Deka, S. Biswas, and B. Bhattacharya, "On-line detection and diagnosis of stuck-at faults in channels of NOC-based systems," in 2016 IEEE International Conference on Systems, Man, and Cybernetics, October 2016, pp. 004567 - 004572. 


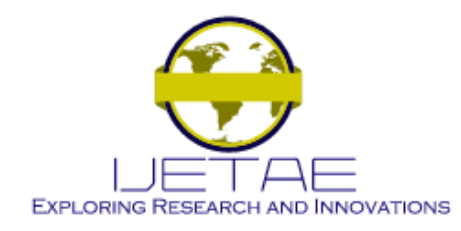

\section{International Journal of Emerging Technology and Advanced Engineering Website: www.ijetae.com (ISSN 2250-2459, ISO 9001:2008 Certified Journal, Volume 10, Issue 07, July 2020)}

[16] G. S. M. Wirthlin, D. Lee and H. Quinn, "Method and case study on identifying physically adjacent multiple-cell upsets using 28-nm, interleaved and SECDEC-protected arrays," in IEEE Trans. Nuclear Science, vol. 61, no. 6, December 2014, pp. 3500-3505.

[17] L. A. Tambara, J. Tonfat, F. L. Kastensmidt, N. H. Medina, N. Added, V. A. P. Aguiar, F. Aguirre, and M. A. G. Silveira, "Analyzing reliability and performance tradeoffs of HLS-based designs in SRAM-based FPGAs under soft errors," in IEEE Transactions on Nuclear Science, 2017, pp. 1-8.

[18] D. Jinling and X. Aiqiang, "A fault simulation method based on mutated truth table of logic gates," in 2016 IEEE International Conference on Integrated Circuits and Microsystems, 2016, pp. 28 32.

[19] R. Bishnoi, F. Oboril, and M. Tahoori, "Fault tolerant non-volatile spintronic flip-flop," in 2016 Design, Automation \& Test in Europe Conference \& Exhibition (DATE), 2016, pp. 261-264.

[20] L. Cassiano, A. Bosio, and G. Natale, "A novel adaptive fault tolerant flip-flop architecture based on tmr," in 2014 19th IEEE European Test Symposium (ETS), 2014, pp. 1-2.

[21] Y. Lin, M. Zwolinski, and B. Halak, "A low-cost radiation hardened flip-flop," in 2014 Design, Automation \& Test in Europe Conference \& Exhibition (DATE), 2014, pp. 1-6.

[22] C. Hu, Y. Du, J. Chen, Y. Chi, and H. Chen, "Novel time redundant flip-flop structure to attenuate the single-event transient," in 2016 13th IEEE International Conference on Solid-State and Integrated Circuit Technology (ICSICT), 2016, pp. 1-3.

[23] V. Bonato, "Máquinas de Estado," 2017, Available in: <http://wiki.icmc.usp.br/images/7/7d/Aula 5 - StateMachine.pdf>.

[24] M. Amagasaki, Y. Nakamura, T. Teraoka, M. Iida, and T. Sueyoshi, "A novel soft error tolerant FPGAarchitecture," in 2016 IFIP/IEEE International Conference on Very Large Scale Integration (VLSISoC), 2016, pp. 1-6.

[25] S. Mandal, R. Paul, S. Sau, A. Chakrabarti, and S. Chattopadhyay, "A novel method for soft error mitigation in FPGA using modified matrix code," in IEEE EMBEDDED SYSTEMS LETTERS, vol. 8, no. 4, Dec 2016, pp. 65-68.

[26] E. Hallett, G. Corradi, and S. McNeil, "Xilinx reduces risk and increases efficiency for iec61508 and iso26262 certified safety applications," Xilinx, Tech. Rep., April 2015.

[27] A. Drozd, I. Perebeinos, O. Martynyuk, K. Zashcholkin, O. Ivanova, and M. Drozd, "Hidden fault analysis of fpga projects for critical applications," in 2020 IEEE 15th International Conference on Advanced Trends in Radioelectronics, Telecommunications and Computer Engineering (TCSET), Feb 2020.

[28] A. Drozd, M. Kuznietsov, A. Antoshchuk, A. Martynyuk, and J. Sulima, "Evolution of a problem of the hidden faults in the digital components of safety-related systems," in 2018 IEEE East-West Design Test Symposium (EWDTS), Nov 2018.

[29] O. Drozd, M. Kuznietsov, O. Martynyuk, and M. Drozd, "A method of the hidden faults elimination in fpga projets for the critical applications," in The 9th IEEE International Conference on Dependable Systems, Services and Technologies, DESSERT2018, May 2018, pp. 218-221.

[30] B. Jeppesen, M. Rajamani, and K. Smith, "Enhancing functional safety in FPGA-based motor drives," in The 9th International Conference on Power Electronics, Machines and Drives (PEMD 2018), vol. 2019, Feb 2019, pp. 4580-4584.
[31] T. Instruments, "Rm48x 16/32-bit RISC flash microcontroller," 2013.

[32] S. Venkataraman, R. Santos, and A. Kumar, "A flexible inexact TMR technique for SRAM-based FPGAs," in 2016 Design, Automation \& Test in Europe Conference \& Exhibition (DATE), 2016, pp. 810-813.

[33] E. Gracic, A. Hayek, and J. Börcsök, "Approach to the development of an FPGA-based safety-related, complete communication computer system," in 2014 X International Symposium on Telecommunications (BIHTEL), October 2014, pp. 1-6.

[34] A. Hayek and J. Börcsök, "SRAM-based FPGA design techniques for safety related systems conforming to IEC 61508," in 2012 2nd International Conference on Advances in Computational Tools for Engineering Applications (ACTEA), 2012, pp. 319-324.

[35] A. Bernardini, W. Ecker, and U. Schlichtmann, "Efficient handling of the fault space in functional safety analysis utilizing formal methods," in 2016 IFIP IEEE International Conference on Very Large Scale Integration (VLSI-SoC), 2016, pp. 1-7.

[36] R. Dobler, S. Cechin, T. Weber, and J. Netto, "A software fault injector to validate implementations of a safety communication protocol," in 2016 Seventh Latin-American Symposium on Dependable Computing (LADC), jun 2016, pp. 35-42.

[37] G. H. Lopes, “Análise de sensibilidade na determinação do nível de integridade de segurança," Trabalho de Diplomação em Engenharia Química, Departamento de Engenharia Química, Universidade Federal do Rio Grande do Sul, Porto Alegre, 2010.

[38] A. P. A. V. Melo, "Nível de Integridade de Segurança (SIL) integrado com fatores humanos e organizacionais," Mestrado em Tecnologia de Processos Químicos e Bioquímicos, Escola de Química, Universidade Federal do Rio de Janeiro, Rio de Janeiro, 2012.

[39] Functional safety of electrical/electronic/programmable electronic safety-related systems - Part 3: Software Requirements, International Electrotechnical Comission, Geneva, Switzerland, 2010.

[40] E. Gracic, A. Hayek, and J. B"orcs"ok, "Evaluation of FPGAdesign tools for safety systems with on-chip redundancy referring to the standard IEC 61508," in 2017 2nd International Conference on System Reliability and Safety, Feb 2017, pp. 386-390.

[41] O. Odarushchenko, O. Strjuk, Y. Bulba, K. Leontiiev, A. Ivasyuk, and V. Kharchenko, "Fault insertion software and hardware testing for safety plc-based system SIL certification," in The 9th IEEE International Conference on Dependable Systems, Services and Technologies, may 2018, pp. 202-206.

[42] R. Girardey, M. Hubner, and J. Becker, "Safety aware place and route for on-chip redundancy in safety critical applications," in 2010 IEEE Annual Symposium on VLSI, Feb 2010, pp. 76-82.

[43] — - "Qualification of hardware description language designs for safety critical applications in nuclear power plants," in IEEE TRANSACTIONS ON NUCLEAR SCIENCE, vol. 67, no. 03, Mar 2020, pp. 502-507.

[44] E. Gracic, A. Hayek, and J. Börcsök, "Implementation of a faulttolerant system using safety-related Xilinx tools conforming to the standard IEC 61508," in 2016 International Conference on System Reliability and Science, 2016, pp. 78-83.

[45] Functional safety of electrical/electronic/programmable electronic safety-related systems - Part 2: Requirements for electrical/electronic/programmable electronic safety- related systems, International Electrotechnical Comission, Geneva, Switzerland, 2010. 


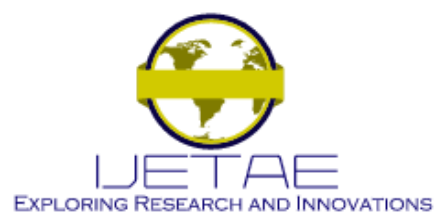

International Journal of Emerging Technology and Advanced Engineering

Website: www.ijetae.com (ISSN 2250-2459, ISO 9001:2008 Certified Journal, Volume 10, Issue 07, July 2020)

[46] Functional safety of electrical/electronic/programmable electronic safety-related systems - part 6: guidelines on the application of IEC 61508-2 and IEC 61508-3 (IEC 61508), International Electrotechnical Comission, Geneva, Switzerland, 2010.

[47] J. Börksök, A. Hayek, and M. Umar, "Implementation of a 1002 RISC architecture on FPGA for safety systems," in 2008 IEEE ACS International Conference on Computer Systems and Applications (AICCSA), 2008, pp. 1046-1051.

[48] I. Siqueira, Manutenção Centrada na Confiabilidade - Manual de Implementação. QualityMark, 2005.
[49] Functional safety of electrical/electronic/programmable electronic safety-related systems - Part 5: Examples of methods for the determination of safety integrity levels, International Electrotechnical Comission, Geneva, Switzerland, 2010.

[50] Functional safety of electrical/electronic/programmable electronic safety-related systems - Part 1: General Requirements, International Electrotechnical Comission, Geneva, Switzerland, 2010. 